\title{
Latest developments and emerging treatment options in the management of stomach cancer
}

This article was published in the following Dove Press journal:

Cancer Management and Research

I2 July 201 I

Number of times this article has been viewed

\section{Thierry Delaunoit}

Medical Oncology Department, Jolimont Hospital, Haine-Saint-Paul, Belgium
Correspondence: Thierry Delaunoit Hôpital de Jolimont, Service d'Oncologie Médicale, Rue Ferrer I59, 7I00

Haine-Saint-Paul, Belgium

Tel +32 64234197

Fax +3264233844

Email thierry.delaunoit@

entitejolimontoise.be

\begin{abstract}
Gastric cancer remains a significant health burden worldwide. Most of these malignancies are diagnosed at an advanced stage and are associated with a grim prognosis. Complete removal of macroscopic and microscopic tumor masses along with regional lymphnodes (R0 surgical resection) represents the treatment of choice in localized, nonmetastatic gastric cancer. Chemotherapy, either alone as a perioperative treatment, or in combination with radiation therapy in an adjuvant setting, improves the clinical outcome for patients with resectable tumors. In patients suffering from metastatic disease, chemotherapy and the so-called targeted therapies play a major role in improving survival and quality of life compared with best supportive care. The emergence of new drugs as well as new administration schedules allow physicians to obtain an objective response of up to $60 \%$ and, since the utilization of targeted therapies, overall survival has reached 14 months. In order to situate the standard of care and the latest developments in gastric malignancies better, the pertinent English literature, including major Phase III randomized studies and meta-analyses, has been reviewed.
\end{abstract}

Keywords: gastric cancer, chemotherapy, radiation therapy, targeted therapies

\section{Introduction}

Despite major efforts to detect upper gastrointestinal malignancies as early as possible, gastric cancer remains one of the leading causes of cancer-related death. It also represents the fourth most commonly diagnosed tumor worldwide, with approximately one million newly detected cancers per year. ${ }^{1,2}$ Small lesions are usually asymptomatic and detected fortuitously or in screening programs, explaining why approximately $65 \%$ of patients are diagnosed in stages III-IV, $85 \%$ with positive lymphonodular involvement and 30\% already with liver metastases. ${ }^{3}$ An additional causative factor explaining the grim prognosis comes from tumor biology and the change in the distribution of gastric cancer from the antrum and body to the proximal stomach. Proximal lesions are in fact biologically more aggressive and have a worse prognosis (stage for stage) than distal gastric cancers. ${ }^{4}$ Less than $50 \%$ of patients are suitable for surgery, which is the only curative modality. According to certain criteria, early gastric cancers limited to the mucosa or submucosa are indicated for endoscopic mucosal resection, while gastrectomy is indicated for more advanced tumors, or as a salvage therapy. The questions of resection type, extent of lymph node dissection, and indication for splenectomy could be debated.

For many years, there were very few reported improvements in the efficacy of chemotherapy for advanced gastric cancer, with median overall survival remaining below 1 year. The emergence of new agents, such as targeted therapies, allows 
physicians to increase survival to around 14 months, for the first time. However, choice of the optimal chemotherapy regimen should take into account the high toxicity of most of the chemotherapy regimens used in this setting and the importance of assessing which patients will benefit the most from treatment, in order to extend the length of life with preservation of quality of life.

This review summarizes the emergent treatments for gastric cancer, based on the standard of care published in the literature based on randomized controlled studies and metaanalyses, in order to help physicians in their clinical practice. A systematic search of all the English language literature regarding gastric cancer treatment was performed, using a MEDLINE search for the period January 2005 to March 2011. Future drug developments are also discussed.

\section{Chemotherapy with curative intent: neoadjuvant and adjuvant treatments}

\section{Perioperative therapy}

Chemotherapy and radiation therapy play a major role in the management of resectable gastric tumors. The combination of different chemotherapeutic drugs in the perioperative setting has been demonstrated to be more effective in resectable gastric cancer than surgery alone. Cunningham et al in a randomized Phase III study comparing surgery alone vs perioperative chemotherapy (epirubicin $50 \mathrm{mg} / \mathrm{m}^{2}+$ cisplatin $60 \mathrm{mg} / \mathrm{m}^{2}$ on day 1 combined with continuous intravenous infusion of 5-FU $200 \mathrm{mg} / \mathrm{m}^{2} /$ day for 21 days $[\mathrm{ECF}])$ plus surgery, demonstrated a statistically significant improvement in progression-free survival and overall survival in the chemotherapy group (Table 1). Interestingly, a noteworthy downstaging effect of chemotherapy was also demonstrated in $\mathrm{T} 1$ and $\mathrm{T} 2$ tumors at surgery in favor of chemotherapy of $51.7 \%$ vs $36.8 \% .{ }^{5}$ Despite the fact that more than one-third of the patients did not start adjuvant chemotherapy, patients assigned to perioperative chemotherapy had a significant survival advantage over those who underwent surgery alone, questioning the role of adjuvant ECF in that setting. The perioperative strategy was also supported by data from the Federation Nationale des Centres de Lutte Contre le Cancer ACCORD07-FFCD 9703 trial of perioperative 5-FU + cisplatin (two or three preoperative cycles of cisplatin $100 \mathrm{mg} / \mathrm{m}^{2}$ on day 1 , and a continuous intravenous infusion of $5-\mathrm{FU} 800 \mathrm{mg} / \mathrm{m}^{2}$ for 5 consecutive days every 28 days), and three or four postoperative cycles of the same regimen for patients with resectable adenocarcinoma of the stomach, esophagogastric junction, or lower esophagus. The reported 5-year overall survival was significantly higher in the chemotherapy group. A better 5-year disease-free survival and a higher R0 resection rate (significantly increased from $73 \%$ to $84 \%$ by neoadjuvant chemotherapy with $P=0.04$ ) were also observed (Table 1). ${ }^{6}$ Based upon these two studies, we can recommend perioperative chemotherapy in resectable gastric tumors, regardless of stage.

\section{Neoadjuvant therapy}

Results with neoadjuvant-only chemotherapy are disappointing. Schumacher et al recently reported the results of a randomized Phase III trial comparing surgery alone with neoadjuvant chemotherapy (cisplatin $50 \mathrm{mg} / \mathrm{m}^{2}$ days 1-15-29, folinic acid $500 \mathrm{mg} / \mathrm{m}^{2}$ and continuous infusion $5-\mathrm{FU} 2 \mathrm{~g} / \mathrm{m}^{2}$ over 24 hours on days 1-8-15-22-29-36) plus surgery. The R0 resection rate was significantly increased by the neoadjuvant chemotherapy $(81.9 \%$ vs $66.7 \%, P=0.036)$, but this study failed to demonstrate any survival advantage in the chemotherapy group (Table 1). ${ }^{7}$ This trial was considered to be statistically under powered to detect a potential survival difference. It is of interest that more than $50 \%$ of patients received reduced or incomplete chemotherapy cycles, which could partly explain the negative results of the study. Other accurate data regarding neoadjuvant therapy are lacking in the literature.

\section{Adjuvant therapy}

Chemotherapy has been widely studied in Japan in the adjuvant setting, especially using the new fluoropyrimidine oral compounds, uracil-tegafur and S-1. In the earliest study, patients were randomized to receive uracil-tegafur vs observation. The 5 -year overall survival was $73 \%$ in the control arm and $86 \%$ in the treatment arm $(P=0.017){ }^{8}$ The study was dedicated to a specific tumor population (pT2pN1-2 adenocarcinoma), rendering the results rather robust (Table 1).

A second positive study was published by Sakuramoto et al in a more heterogeneous group of patients, including all tumor stages (except $\mathrm{T} 1$ lesions). The 3-year overall survival rate was higher in the S-1 group (Table 1). Relapse-free survival at 3 years was also ameliorated by administration of S-1 $(P<0.001) .{ }^{9}$ Based on these results, $\mathrm{S}-1$ has become a standard option in Japan for adjuvant chemotherapy. Numerous randomized clinical trials have compared surgery alone with adjuvant chemotherapy, but definitive evidence is lacking. Many of these trials have had limited sample sizes, making it difficult to draw definitive conclusions. A recently 
published meta-analysis of 17 randomized controlled trials of adjuvant chemotherapy in gastric cancer demonstrated a modest but statistically significant benefit associated with fluorouracil based adjuvant chemotherapy after curative resection of gastric cancers, in terms of overall survival (hazards ratio [HR]: 0.82, 95\% confidence interval [CI]: $0.76-0.90 ; P<0.001$ ) and disease-free survival (HR: 0.82 , 95\% CI: $0.75-0.90 ; P<0.001)$ compared with surgery alone. ${ }^{10}$ This approach might therefore become an alternative in this setting.

Although widely criticized for the quality of the surgery, the Intergroup 0116 trial included a total of 556 patients with completely resected adenocarcinoma of the stomach or esophagogastric junction. They were randomized to receive either adjuvant 5-FU + leucovorin in combination with radiation therapy or observation. The median overall survival was significantly improved in the adjuvant arm (Table 1). Although better in terms of efficacy, the chemoradiation arm was associated with significant toxicity (three toxic deaths [1\%], $41 \%$ grade 3 toxicity, and $32 \%$ grade 4 toxicity). ${ }^{11}$ However, this regimen remains an option in patients for whom preoperative chemotherapy has not been given and when the tumor is at high risk of recurrence.

\section{Palliative setting}

While the use of chemotherapy is indisputable in a palliative setting, treatment decisions should take into account the possible high toxicity commonly observed in most chemotherapy regimens utilized, and balance that toxicity against the benefit to patients. To help clinicians determine good candidates for palliative chemotherapy, Chau et al recently demonstrated that not all patients will benefit from such treatment. They identified independent prognostic factors reducing survival in treated patients, such as a performance status of 2, presence of liver and/or peritoneal involvement, and alkaline phosphatase $>100 \mathrm{U} / \mathrm{L} .{ }^{12}$ Patients with none of these factors benefited from chemotherapy, with a median overall survival of 11.8 months, while patients having three of four prognostic factors had a worse prognosis, with a median survival of 4.1 months. This observation may help physicians to select patients for whom chemotherapy might be beneficial.

\section{Chemotherapy compared with best supportive care}

The majority of the patients with gastric cancer present with advanced disease at diagnosis, rendering the prognosis extremely poor. In these patients, palliative treatments are given with the intention of improving quality of life and prolonging survival. The role of palliative chemotherapy in improving survival compared with best supportive care is no longer controversial, because the results of three studies published 20 years ago demonstrated a clear advantage for patients receiving chemotherapy, in terms of improving either quality of life or survival. ${ }^{13-15}$ Furthermore, data from eleven randomized trials that included approximately 1500 patients from several European and US centers were examined by Wagner et al in a meta-analysis published in 2006 . In the majority of the studies, patients in the control arms received 5-FU-based chemotherapy, administered either as a bolus or as a continuous infusion. Examining the individual studies, nine of eleven did not show significant differences in survival between the two groups. However, the meta-analysis found a statistically significant advantage in favor of combination compared with single-agent chemotherapy (HR: 0.83 , 95\% CI: $0.74-0.93 ; P<0.001) .{ }^{16}$ Quality of life was only studied in one of the eleven trials and demonstrated a real benefit for treated patients, confirming the results of the previous published trials. ${ }^{16}$

\section{Which chemotherapy for first-line therapy?}

Many drugs have been studied in gastric cancer either in combination or as monotherapy. Over the years, we have learnt from the literature that combination therapies are more effective in terms of response rate, overall survival, and quality of life than single drugs, leading to single-agent therapy not being prescribed nowadays in advanced gastric cancer. ${ }^{16}$ We have also observed over the years that two of the oldest drugs, cisplatin and 5-FU, are still worthwhile, and are considered as the cornerstone of many combination therapies.

Physicians have now isolated several chemotherapeutic regimens which can still be used for advanced gastric malignancies (Table 2). Dual therapy of cisplatin +5 -FU was compared with 5-FU + adriamycin + methotrexate and etoposide + leucovorin +5 -FU in a randomized Phase III study published by Vanhoefer et al in 2000. Although the combination did not show any survival advantage, the dual therapy was associated with a significant better response rate and toxicity profile than the two other regimens, showing that dual therapy could be better than multiple drug therapy. ${ }^{17}$ Waters et al published the final results of a randomized Phase III trial comparing ECF with 5-FU + adriamycin + methotrexate. ECF showed a survival advantage compared with 5-FU + adriamycin + methotrexate, with a better overall response 
Table I Neoadjuvant and adjuvant therapy in locally advanced gastric cancer

\begin{tabular}{|c|c|c|c|c|c|c|c|}
\hline & $\mathbf{n}$ & Setting & Arm & DFS (\%) & $P$ value & OS (\%) & $P$ value \\
\hline Cunningham et $\mathrm{a}^{5}$ & $250 / 253$ & Perioperative & $\mathrm{POC} / \mathrm{S}$ & $30 / 18$ & $<0.001$ & $36.3 / 29.5$ & $=0.009$ \\
\hline Ychou et al $\left.\right|^{6}$ & $113 / 111$ & Perioperative & $\mathrm{POC} / \mathrm{S}$ & $34 / 19$ & $=0.003$ & $38 / 24$ & $=0.021$ \\
\hline Schuhmacher et $\mathrm{al}^{7}$ & $72 / 72$ & Neoadjuvant & $\mathrm{NC}+\mathrm{S} / \mathrm{S}$ & - & NS & - & NS \\
\hline Nakajima et $\mathrm{al}^{8}$ & $95 / 95$ & Adjuvant & $\mathrm{S}+\mathrm{UFT} / \mathrm{S}$ & - & - & $86 / 73$ & 0.017 \\
\hline Sakuramoto et al ${ }^{9}$ & $529 / 530$ & Adjuvant & $S+S-I / S$ & - & - & $80.1 / 70.1$ & 0.003 \\
\hline MacDonald et al" & $28 I / 275$ & Adjuvant & $\mathrm{S}+\mathrm{CR} / \mathrm{S}$ & $48 / 31$ & $<0.001$ & $50 / 41$ & 0.005 \\
\hline
\end{tabular}

Abbreviations: DFS, disease-free survival; OS, overall survival; POC, perioperative chemotherapy; S, surgery; NC, neoadjuvant; CR, chemoradiotherapy.

rate and toxicity profile (Table 2).$^{18}$ This combination is still in use in many countries, but has been progressively replaced by new combination therapies of docetaxel + cisplatin +5 -FU, although no head-to-head comparisons have been reported in the literature demonstrating that docetaxel + cisplatin +5 -FU is better than ECF. The only relevant data published in the literature comes from a randomized Phase II trial studying three chemotherapy regimens, ie, docetaxel + cisplatin + 5-FU, docetaxel + cisplatin, and ECF. The authors showed a trend favoring the docetaxel + cisplatin +5 -FU combination in terms of response rate and median time to progression compared with ECF. However, despite its apparent lower efficacy, the ECF regimen was better tolerated, with patients experiencing less toxicity and having a better quality of life..$^{19}$ It is of interest to note that this randomized Phase II study was not designed to compare the three regimens with each other.

Docetaxel $75 \mathrm{mg} / \mathrm{m}^{2}+$ cisplatin $75 \mathrm{mg} / \mathrm{m}^{2}+5-\mathrm{FU}$ $750 \mathrm{mg} / \mathrm{m}^{2}$ on days $1-5$ every 3 weeks has recently become the standard of care in metastatic gastric tumors, and improved response rate, progression-free survival, and overall survival compared with cisplatin +5 -FU as the control treatment in a randomized Phase III trial published by Van
Cutsem et al (Table 2). ${ }^{20}$ One should keep in mind that the percentage of patients receiving subsequent lines of treatment was lower for the docetaxel + cisplatin +5 -FU arm than for the cisplatin +5 -FU arm (32\% vs $41 \%)$, rendering the survival results rather robust. Despite high hematological toxicity (grade 3-4 neutropenia in $82 \%$, complicated neutropenia in 29\%), the authors demonstrated that quality of life was better preserved in the docetaxel + cisplatin +5 -FU arm. ${ }^{21}$ Physicians have tried to improve the toxicity profile of the standard docetaxel + cisplatin +5 -FU regimen by either using a different administration schedule or modifying the combination itself. ${ }^{22-24}$ Data from 95 patients treated with weekly docetaxel $20 \mathrm{mg} / \mathrm{m}^{2}+$ cisplatin $20 \mathrm{mg} / \mathrm{m}^{2}+5$-FU $350 \mathrm{mg} / \mathrm{m}^{2}$ for 6 consecutive weeks followed by a 2-week break were retrospectively collected from 2002 to 2006 at The University of Texas MD Anderson Center. A response rate of $34 \%$ (95\% CI: $24-45)$ and a median overall survival of 8.9 months $(95 \%$ CI: $7.7-10.8)$ were observed. ${ }^{22}$ Hematological toxicity was tolerable. However, caution is advised when interpreting the results regarding retrospective analysis of the data. In a randomized Phase II trial, Tebbutt et al studied a weekly regimen of docetaxel $30 \mathrm{mg} / \mathrm{m}^{2}$ on

Table 2 Results of major Phase III studies using combination therapies in first-line therapy for metastatic gastric cancer

\begin{tabular}{|c|c|c|c|c|c|c|c|c|c|}
\hline & $\mathbf{n}$ & Regimen & RR (\%) & TTF & $P$ value & $\begin{array}{l}\text { Median } \\
\text { PFS }\end{array}$ & $P$ value & $\begin{array}{l}\text { Median } \\
\text { OS }\end{array}$ & $P$ value \\
\hline Waters et al $^{18}$ & $130 / 126$ & FAMTX/ECF & $21 / 46$ & - & - & - & - & $6.1 / 8.7$ & 0.0005 \\
\hline Vanhoefer et $\mathrm{al}^{17}$ & $134 / 132 / 133$ & CF/ELF/FAMTX & $20 / 9 / 12$ & - & - & $4.1 / 3.3 / 3.3$ & NS & 7.2/7.2.6.9 & NS \\
\hline Van Cutsem et $\mathrm{al}^{20}$ & $224 / 221$ & $\mathrm{CF} / \mathrm{DCF}$ & $25 / 37$ & - & - & $3.7 / 5.6$ & $<0.001$ & $8.6 / 9.2$ & 0.02 \\
\hline Cunningham et $\mathrm{al}^{27}$ & $\begin{array}{l}263 / 245 / \\
250 / 244\end{array}$ & $\begin{array}{l}\text { ECF/EOF/ } \\
\text { ECX/EOX }\end{array}$ & $4 I / 42 / 46 / 48$ & - & - & $6.2 / 6.5 / 6.7 / 7$ & NS & $\begin{array}{l}9.9 / 9.3 / 9.9 / \\
11.2\end{array}$ & 0.02 \\
\hline Dank et al ${ }^{30}$ & $172 / 165$ & $\mathrm{IF} / \mathrm{CF}$ & $31.8 / 25.8$ & $4 / 3.4$ & 0.0018 & $7.4 / 7.6$ & NS & $9 / 8.7$ & NS \\
\hline Guimbaud et a $\left.\right|^{31}$ & $174 / 175$ & ECX/ FOLFIRI & & $4 / 5$ & 0.008 & $5.3 / 5.75$ & - & $9.5 / 9.7$ & NS \\
\hline Boku et al ${ }^{32}$ & $234 / 235 / 234$ & 5-FU/CP/S-I & $9 / 38 / 28$ & - & - & - & - & $\begin{array}{l}\text { I0.8/I2.3/ } \\
11.4\end{array}$ & NS \\
\hline Kang et $\mathrm{al}^{34}$ & $160 / 156$ & XP/FP & $46 / 32$ & - & - & $5.6 / 5$ & NS & $10.5 / 9.3$ & NS \\
\hline Koizumi et $\mathrm{al}^{35}$ & $148 / 150$ & CS-I/S-I & $54 / 31$ & - & - & $6 / 4$ & $<0.000 \mathrm{I}$ & $|3 /| \mid$ & 0.04 \\
\hline Ajani et $\mathrm{al}^{36}$ & $527 / 526$ & $\mathrm{CS}-\mathrm{I} / \mathrm{CF}$ & $29.1 / 31.9$ & - & - & $4.8 / 5.5$ & NS & $8.6 / 7.9$ & NS \\
\hline
\end{tabular}

Abbreviations: $n$, number of patients; NS, not significant; RR, response rate; TTF, time to treatment failure; PFS, progression free survival; OS, overall survival; $F$, fluorouracil; 5-FU, infusional 5-fluorouracil; A, adriamycin; MTX, methotrexate; E, epirubicin; C, cisplatin; L, leucovorin; D, docetaxel; O, oxaliplatin; X, capecitabine; I or IRI, irinotecan. 
days 1 and $8+$ cisplatin $60 \mathrm{mg} / \mathrm{m}^{2}$ on day $1+5$-FU $200 \mathrm{mg} /$ $\mathrm{m}^{2} /$ day continuously, every 3 weeks, and a combination of docetaxel $30 \mathrm{mg} / \mathrm{m}^{2}$ on days 1 and $8+$ capecitabine $1600 \mathrm{mg} / \mathrm{m}^{2} /$ day on days $1-14$, every 3 weeks. Response rates were $47 \%$ and $26 \%$ in the weekly docetaxel + cisplatin + 5-FU and the docetaxel + capecitabine arm, respectively, whereas a better median overall survival was observed in the weekly docetaxel + cisplatin + 5-FU arm (11.2 months and 10.1 months, respectively). Both groups showed a favorable toxicity profile, especially in terms of hematological toxicity. ${ }^{23}$ Subsequently, Li et al compared the combination of weekly docetaxel $36 \mathrm{mg} / \mathrm{m}^{2}+$ cisplatin $30 \mathrm{mg} / \mathrm{m}^{2}$ on days 1 and 8 with uracil-tegafur $300 \mathrm{mg} / \mathrm{m}^{2} /$ day on days 1-14 every 3 weeks in 45 chemo-naïve patients suffering from metastatic gastric cancer. The results were rather interesting because the response rate reached $58 \%$, with a median overall survival of 13.9 months, which is rarely observed in this setting. The toxicity profile was also tolerable. ${ }^{24}$ These data suggest that ameliorating the toxicity profile of the docetaxel + cisplatin +5 -FU regimen is feasible without jeopardizing efficacy. However, Phase III trials are needed to confirm this approach.

Oxaliplatin, a new platinum compound, and irinotecan, a topoisomerase I inhibitor, both of which are active in colorectal cancer, ${ }^{25,26}$ have brought new hope in gastric cancer management. Oxaliplatin has been combined with epirubicin and 5-FU or capecitabine in a noninferiority randomized Phase III study (REAL-2) detailed in Table $2 .{ }^{27}$ On day 1 of every 3 -week cycle, patients in all study groups received an intravenous bolus of epirubicin $50 \mathrm{mg} / \mathrm{m}^{2}$, cisplatin $60 \mathrm{mg} / \mathrm{m}^{2}$ was given intravenously with hydration in the ECF and epirubicin + cisplatin + capecitabine groups, and oxaliplatin $130 \mathrm{mg} / \mathrm{m}^{2}$ was administered intravenously over a 2-hour period in the epirubicin + oxaliplatin $+5-\mathrm{FU}$ and epirubicin + oxaliplatin + capecitabine groups. 5 -FU $200 \mathrm{mg} / \mathrm{m}^{2}$ and capecitabine $625 \mathrm{mg} / \mathrm{m}^{2}$ twice daily were given throughout treatment. Median overall survival was in favor of the epirubicin + oxaliplatin + capecitabine group (HR for death 0.80 in the epirubicin + oxaliplatin + capecitabine group $(95 \% \mathrm{CI}$ : $0.66-0.97 ; P=0.02)$. Oxaliplatin was associated with less alopecia, and hematological and renal toxicity than cisplatin, whereas no difference was seen in terms of toxicity between 5-FU and capecitabine. Based upon these results, oxaliplatin has a better toxicity profile than cisplatin without jeopardizing efficacy. Two promising Phase I trials have been recently published combining docetaxel + oxaliplatin + capecitabine. Although the dose-limiting toxicity was different between the two studies, the combination might potentially become a valuable regimen in metastatic gastric cancer. ${ }^{28,29}$ Further evaluations using this combination are ongoing.

The advent of irinotecan has brought new opportunities for the management of metastatic gastric cancer by enabling use of combinations that do not contain a platinum compound such as oxaliplatin or cisplatin. Randomized Phase III studies have recently been published in the literature. Dank et al compared a weekly regimen of irinotecan $+5-\mathrm{FU}$ (irinotecan $80 \mathrm{mg} / \mathrm{m}^{2}$, folinic acid $500 \mathrm{mg} / \mathrm{m}^{2}, 5-\mathrm{FU}$ $2000 \mathrm{mg} / \mathrm{m}^{2}$ for 6 weeks with 1 week rest) with the traditional cisplatin $+5-\mathrm{FU}$ combination. Although no survival advantage was seen in the irinotecan +5 -FU group, this combination was not inferior to cisplatin $+5-\mathrm{FU}$, with a comparable response rate, progression-free survival, and overall survival (Table 2 ) ${ }^{30}$ Recently presented data from the Federation Nationale des Centres de Lutte Contrele Cancer ACCORD07-FFCD 0307 trial showed encouraging results using FOLFIRI (irinotecan $180 \mathrm{mg} / \mathrm{m}^{2}$ on day 1 , folinic acid $400 \mathrm{mg} / \mathrm{m}^{2}$ day 1 , bolus 5 -FU $400 \mathrm{mg} / \mathrm{m}^{2}$ on day 1 and continuous infusion of 5-FU $2400 \mathrm{mg} / \mathrm{m}^{2}$ on days 1 and 2 , every 2 weeks) compared with epirubicin + cisplatin + capecitabine (epirubicin $50 \mathrm{mg} / \mathrm{m}^{2}$ on day $1+$ cisplatin $60 \mathrm{mg} / \mathrm{m}^{2}$ on day $1+$ capecitabine $2000 \mathrm{mg} / \mathrm{m}^{2}$ on days $2-15$, every 3 weeks). Both combinations showed the same results in terms of progression-free survival and overall survival, the toxicity profile being more favorable in the FOLFIRI arm. ${ }^{31}$ A new parameter, firstly studied by Dank et al, ie, the time to therapeutic failure (corresponding to the time between randomization and progression, treatment discontinuation, recurrence, or death) was significantly improved in the FOLFIRI arm compared with epirubicin + cisplatin + capecitabine (5 months vs 4 months, HR: 0.77, CI: 95\% 0.63-0.94; $P=0.008)$. These studies show that a noncisplatin-based treatment can have equivalent efficacy to a cisplatin-based combination in advanced gastric tumors, with fewer serious adverse events and treatment withdrawals because of toxicity. Therefore, dual therapy of irinotecan +5 -FU is a useful alternative for patients who cannot receive a cisplatin-based regimen and might become a new standard in first-line therapy for metastatic gastric cancer. The Japan Clinical Oncology Group 9912 trial randomized more than 700 patients with advanced disease to either a cisplatinirinotecan doublet or single-agent treatment with infusional 5-FU or the new oral fluoropyrimidine, S-1 (Table 2). The cisplatin-irinotecan doublet achieved a superior response rate but failed to demonstrate any advantage in terms of 
overall survival compared with the single-agent 5-FU treatment, with a rather interesting 12.3 months of median overall survival $(P=0.055)$. However, in this patient population, the combined regimen was considerably more toxic than the monotherapy regimen and was associated with relatively high rates of toxicity-related withdrawal from treatment. The most common grade 3 toxicity was neutropenia (65\% in the cisplatin + irinotecan arm compared with approximately $15 \%$ in the 5 -FU and S-1 arms), with a $9 \%$ incidence of febrile neutropenia. Nonhematological toxicities were also markedly higher in the doublet than in the monotherapy arms. ${ }^{32}$ Therefore, this combination is not recommended as a first choice and must be used with caution.

Oral 5-FU has been developed in order to avoid the need for a central venous catheter which is inconvenient for patients and potentially associated with morbidity. Capecitabine has been shown to be noninferior to infusional 5-FU in doublet and triplet regimen studies. ${ }^{27,33}$ The doublet of capecitabine + cisplatin was compared with cisplatin +5 -FU in 316 chemo-naïve patients suffering from advanced gastric adenocarcinoma (Table 2). The median progression-free survival was identical between the groups (5.6 and 5.0 months for the capecitabine + cisplatin and cisplatin +5 -FU arms, respectively), thus meeting the primary objective of the study. The toxicity profile was similar between the groups, apart from hand-foot syndrome, which was higher in the capecitabine arm, as expected from other studies. ${ }^{34}$ Noninferiority in relation to survival with capecitabine vs 5-FU in triplet regimens for the treatment of patients with advanced esophagogastric cancer was also demonstrated in the REAL-2 trial (HR: 0.86, 95\% CI: $0.80-0.99)$, as described in Table 2 and previously discussed. ${ }^{27}$

S-1, mainly developed in Japan, is a combination of three pharmacological components, ie, tegafur (a prodrug of 5-FU), 5-chloro-2,4 dihydropyridine (a powerful inhibitor of dihydropyrimidine dehydrogenase), and potassium oxonate (to protect against drug-induced diarrhea). The noninferiority of S-1 compared with infusional 5-FU has been demonstrated in the Japan Clinical Oncology Group 9912 trial and does not need further discussion. ${ }^{32}$

The recent SPIRITS trial showed superiority in terms of response rate, progression-free survival (6 vs 4 months, $P<0.0001$ ), and overall survival (13 vs 11 months, $P=0.04$ ) for the doublet regimen combining cisplatin $\left(60 \mathrm{mg} / \mathrm{m}^{2}\right.$ day 8$)$ and S-1 (40-60 mg depending on patient's body surface area, given orally twice daily for 3 consecutive weeks) followed by a 2-week rest, compared with $\mathrm{S}-1$ alone (same doses), leading Asian countries to consider this doublet as a standard for first-line therapy in metastatic gastric cancer ${ }^{35}$ (Table 2). Results from the FLAGS trial developed in Western countries comparing the traditional 5-FU + cisplatin (infusional 5 -FU $1000 \mathrm{mg} / \mathrm{m}^{2} / 24$ hours for 120 hours and cisplatin at $100 \mathrm{mg} / \mathrm{m}^{2}$ intravenously on day 1 , repeated every 28 days) with cisplatin + S-1 (S-1 $50 \mathrm{mg} / \mathrm{m}^{2}$ divided in two daily doses for 21 days and cisplatin at $75 \mathrm{mg} / \mathrm{m}^{2}$ intravenously on day 1 , repeated every 28 days) failed to demonstrate any survival advantage for the experimental group (Table 2). Response rates and progression-free survival, although not statistically significant, were in favor of the cisplatin +5 -FU arm. ${ }^{36}$ These discrepant results led physicians in Western countries not to recommend this combination. However, further analyses are needed to understand the huge differences observed between the studies.

Targeted therapies have emerged as a new hope in cancer management during recent years. Several drugs have been studied in gastric cancer, among which are angiogenesis and human epidermal growth factor receptor 1 and 2 (HER1 and HER2) inhibitors. The first to have demonstrated its efficacy in gastric malignancies is trastuzumab, a monoclonal antibody, targeting HER2. The ToGA trial (Table 2) in fact showed that adjunctive addition of trastuzumab (intravenously at a dose of $8 \mathrm{mg} / \mathrm{kg}$ on day 1 of the first cycle, followed by $6 \mathrm{mg} / \mathrm{kg}$ every 3 weeks) to cisplatin (intravenous infusion $80 \mathrm{mg} / \mathrm{m}^{2}$ on day 1) and 5-FU (intravenous infusion $800 \mathrm{mg} / \mathrm{m}^{2}$ per day on days $1-5$ of each cycle) or capecitabine $(1000 \mathrm{mg} /$ $\mathrm{m}^{2}$ given orally twice a day for 14 days followed by a 1 -week rest) was of benefit for patients with tumors showing overexpression of HER 2 protein by immunohistochemistry or gene amplification by fluorescence in situ hybridization compared with chemotherapy alone. ${ }^{37}$ The combination reached for the first time in Western countries an overall survival above 1 year (Table 3). Median progression-free survival and overall response rate was also ameliorated by trastuzumab (Table 3 ). Apart from diarrhea, the toxicity profile was identical in both groups, and particularly for cardiac adverse events, a specific toxicity observed with trastuzumab therapy, which was quite low with less than $1 \%$ of patients experiencing such problems. Based upon these results, the combination of trastuzumab and chemotherapy has become the standard of care in first-line therapy for patients suffering from HER2-positive gastric neoplasms.

The antiangiogenesis monoclonal antibody, bevacizumab, directed against the vascular endothelial growth factor receptor, 
Table 3 Targeted therapies in metastatic gastric cancer: current results

\begin{tabular}{lllllll}
\hline & Phase & $\mathbf{n}$ & Regimen & RR $(\%)$ & PFS $(\mathbf{m})$ & OS $(\mathbf{m})$ \\
\hline Bang et a| $^{37}$ & III & $298 / 296$ & Tr-C-5-FU/C-5FU & $47 / 35(P=0.0017)$ & $6.7 / 5.5(P=0.0002)$ & $13.8 / 11.1(P=0.0046)$ \\
Kang et al $^{38}$ & III & $387 / 387$ & B-C-5-FU/C-5FU & $38 / 29.5(P=0.0121)$ & $6.7 / 5.3(P=0.0037)$ & $12.1 / 10.1(\mathrm{NS})$ \\
El-Rayes et al $^{39}$ & II & 38 & B-O-D & 42 & 6.6 & 11.1 \\
Sun et al $^{40}$ & II & 44 & Sor-C-D & $4 I$ & 5.8 & 13.5 \\
\hline
\end{tabular}

Abbreviations: $n$, number of patients; RR, response rate; TTF, time to treatment failure; PFS, progression free survival; OS, overall survival; Tr, trastuzumab; C, cisplatin; 5-FU, 5-fluorouracil; B, bevacizumab; O, oxaliplatin; D, docetaxel, Sor, sorafenib.

has, on the other hand, failed to demonstrate any survival advantage in the randomized Phase III AVAGAST trial where the chemotherapy was identical to that used in the ToGA trial. However, median progression-free survival and response rate was significantly improved in the bevacizumab group (Table 3 ), with a favorable safety profile. Interestingly, variability in median overall survival were observed between geographical areas, and especially in the chemotherapy only groups, with a median overall survival of 8.6 and 6.8 months in the US and Europe, respectively. ${ }^{38}$ Mature and definitive data are awaited, especially in terms of second-line therapy used in both groups, which may explain the differences observed in survival.

Replacing cisplatin with oxaliplatin, El-Rayes et al assessed the combination of bevacizumab $7.5 \mathrm{mg} / \mathrm{kg}$, docetaxel $70 \mathrm{mg} / \mathrm{m}^{2}$, and oxaliplatin $75 \mathrm{mg} / \mathrm{m}^{2}$ on day 1 , every 3 weeks in a open-label Phase II study including 38 patients, ${ }^{39}$ and the results are encouraging (Table 3). Sorafenib, a tyrosine kinase inhibitor targeting angiogenesis, has been recently studied in a Phase II study including 44 patients. Sorafenib $400 \mathrm{mg}$ orally twice a day was combined with cisplatin $75 \mathrm{mg} / \mathrm{m}^{2}$ on day 1 and docetaxel $75 \mathrm{mg} / \mathrm{m}^{2}$ on day 1 , and repeated every 21 days. A remarkable median overall survival of 13.5 months was reached, with a tolerable toxicity profile. ${ }^{40}$ This combination deserves further evaluation.

The Phase III EXPAND and REAL-3 trials are currently assessing the role of monoclonal antibodies vs the epidermal growth factor receptor in combination with chemotherapy. Cetuximab is combined with cisplatin and capecitabine in the EXPAND study, whereas panitumumab is combined with the English standard of epirubicin + oxaliplatin + capecitabine in the REAL-3 trial. These studies are ongoing and results are pending.

\section{Place of second-line therapy in metastatic gastric cancer}

There is no huge difference in terms of response rate when comparing patients with colorectal cancer and those with gastric cancer. However, corresponding improvement in median overall survival has not yet been reached by the combination therapies currently available. This lack of progress in improving overall survival is partly due to differences in the surgical management of metastatic disease, and also related to the limited efficacy of second-line and third-line treatments for advanced disease, given that, unlike patients with colorectal cancer, patients suffering from metastatic gastric malignancies are usually not able to receive second-line or third-line chemotherapy. Currently available data for second-line and third-line therapy are emerging from Phase II studies, although one Phase III study has assessed the role of marimastat in first-line and second-line therapy in patients suffering from metastatic gastric tumors. A significant survival benefit was identified at study completion in the subgroup of patients who had received prior chemotherapy (HR: $1.53,95 \% \mathrm{CI}: 1.00$ $0.34 ; P=0.045)$. Furthermore, marimastat did not worsen the prognosis when used as first-line therapy. Median progression-free survival was also significantly longer for patients receiving marimastat compared with placebo (HR: 1.32, 95\% CI: $1.07-1.63 ; P=0.009) .{ }^{41}$ Several Phase II studies assessing different chemotherapeutic regimens in a small number of patients have been published. ${ }^{42-49}$ Response rates are between $11 \%$ and $32 \%$, with median time to progression of around 3 months and a median overall survival reaching 11.7 months in a study of sunitinib, an oral, multitargeted tyrosine kinase inhibitor, as singleagent therapy in 42 evaluable gastric cancer patients with stage IV disease who had failed first-line chemotherapy. The toxicity profile was also favorable. ${ }^{49}$ Therefore, sunitinib deserves our attention and should be further assessed in clinical trials, either alone or in combination with other chemotherapeutic agents.

\section{Conclusion}

Major advances have been made during the last two decades in the management of gastric cancer, not only for metastatic disease but also for resectable disease. The multidisciplinary approach involving the surgeon as well as the radiation thera- 
pist and oncologist allows clinicians to improve survival in patients with locally advanced disease amenable to surgery.

Perioperative chemotherapy and adjuvant chemoradiation are the two options confirmed in the literature to improve the surgical outcome, the latter being reserved for patients not treated by chemotherapy before surgery, and having a high risk of tumor recurrence, based upon pathological findings.

In the case of palliative treatment, the endpoint is to slow tumor growth as far as possible. The currently available firstline treatments for advanced gastric cancer offer a clear survival benefit to patients compared with best supportive care alone. Apart from the doublet of cisplatin + S-1, triple regimens, such as docetaxel + cisplatin +5 -FU, epirubicin + oxaliplatin + capecitabine, and trastuzumab + cisplatin $+5-\mathrm{FU}$, have demonstrated benefit compared with other regimens. Although no direct comparative data suggest which of these is the most effective, the trastuzumab + cisplatin $+5-\mathrm{FU}$ combination seems to be the best regimen for patients expressing HER2, while docetaxel + cisplatin + 5-FU is recommended for HER2-negative patients. Because docetaxel + cisplatin +5 -FU is an intensive combination, some consideration must be given to the use of docetaxel with less intensive regimens (such as with oral fluoropyrimidines or by using weekly schedules). However, recommendations for therapy should be individualized, based on the patient's performance status and comorbidities.

Many oncologists consider all gastric tumors as different entities, either based upon histology or tumor location, and even by molecular markers. It is unclear why certain tumors grow differently when they are similar in terms of histology and location. Better comprehension of the molecular mechanisms responsible for the genesis of gastric cancer is fundamental to improve tumor management. Translational research has already brought hope in gastric cancer, allowing physicians to select tumors responsive to targeted therapies. Future research will surely help clinicians to select and adapt these treatments to individual patients.

However, it is unclear why tumors benefit differently from chemotherapy with regard to their histology or location. Since the majority of the published studies have included patients with tumors located at different sites, with different tumor stage and histology, rendering the populations quite heterogeneous. Future trials might perhaps select patient populations better, taking into account the different parameters cited above, as well as biomarkers already utilized in other malignancies, such as breast cancer.
The future of gastric cancer management should focus not only on the best chemotherapeutic regimen, but also on improving early response evaluation in order to avoid long, toxic, and ineffective treatments, and to find prognostic biomarkers which allow physicians to tailor treatment to the individual. The role of metabolic assessment using positron emission tomography has been proven effective in predicting responders to chemotherapy in terms of histologic tumor regression and patient survival in patients treated with preoperative chemotherapy followed by surgery. ${ }^{50} \mathrm{~A}$ recent review of the literature showed that positron emission tomography can contribute to the selection of a more appropriate treatment modality by better detecting distant metastases and treatment response than conventional imaging techniques. ${ }^{51}$

\section{Disclosure}

The author reports no conflicts of interest in this work.

\section{References}

1. Kamangar F, Dores GM, Anderson WF. Patterns of cancer incidence, mortality, and prevalence across five continents: defining priorities to reduce cancer disparities in different geographic regions of the world. J Clin Oncol. 2006;24(14):2137-2150.

2. Roder DM. The epidemiology of gastric cancer. Gastric Cancer. 2002; 5(Suppl 1):5-11.

3. Adachi Y, Kitano S, Sugimachi K. Surgery for gastric cancer: 10-year experience worldwide. Gastric Cancer. 2001;4(4):166-174.

4. Craanen ME, Dekker W, Blok P, Ferwerda J, Tytgat GN. Time trends in gastric carcinoma: changing patterns of type and location. Am J Gastroenterol. 1992;87(5):572-579.

5. Cunningham D, Allum WH, Stenning SP, et al. Perioperative chemotherapy versus surgery alone for resectable gastroesophageal cancer. N Engl J Med. 2006;355(1):11-20.

6. Ychou M, Boige V, Pignon JP, et al. Perioperative chemotherapy compared with surgery alone for resectable gastroesophageal adenocarcinoma: a FNCLCC and FFCD multicenter phase III trial. J Clin Oncol. 2011;29(13):1715-1721.

7. Schuhmacher C, Gretschel S, Lordick F, et al. Neoadjuvant chemotherapy compared with surgery alone for locally advanced cancer of the stomach and cardia: European Organisation for Research and Treatment of Cancer randomized trial 40954. J Clin Oncol. 2010;28(35):5210-5218.

8. Nakajima T, Kinoshita T, Nashimoto A, et al. Randomized controlled trial of adjuvant uracil-tegafur versus surgery alone for serosa-negative, locally advanced gastric cancer. Br J Surg. 2007;94(12):1468-1476.

9. Sakuramoto S, Sasako M, Yamaguchi T, et al. Adjuvant chemotherapy for gastric cancer with S-1, an oral fluoropyrimidine. $N$ Engl J Med. 2007;357(18):1810-1820

10. Paoletti X, Oba K, Burzykowski T, et al. Benefit of adjuvant chemotherapy for resectable gastric cancer: a meta-analysis. JAMA. 2010; 303(17):1729-1737.

11. Macdonald JS, Smalley SR, Benedetti J, et al. Chemoradiotherapy after surgery compared with surgery alone for adenocarcinoma of the stomach or gastroesophageal junction. $N$ Engl J Med. 2001;345(10):725-730.

12. Chau I, Norman AR, Cunningham D, Waters JS, Oates J, Ross PJ. Multivariate prognostic factor analysis in locally advanced and metastatic esophago-gastric cancer - pooled analysis from three multicenter, randomized, controlled trials using individual patient data. J Clin Oncol. 2004;22(12):2395-2403. 
13. Pyrhonen S, Kuitunen T, Nyandoto P, Kouri M. Randomised comparison of fluorouracil, epidoxorubicin and methotrexate (FEMTX) plus supportive care with supportive care alone in patients with non-resectable gastric cancer. Br J Cancer. 1995;71(3):587-591.

14. Glimelius B, Ekstrom K, Hoffman K, et al. Randomized comparison between chemotherapy plus best supportive care with best supportive care in advanced gastric cancer. Ann Oncol. 1997;8(2):163-168.

15. Murad AM, Santiago FF, Petroianu A, Rocha PR, Rodrigues MA, Rausch M. Modified therapy with 5-fluorouracil, doxorubicin, and methotrexate in advanced gastric cancer. Cancer. 1993;72(1): $37-41$.

16. Wagner AD, Grothe W, Haerting J, Kleber G, Grothey A, Fleig WE. Chemotherapy in advanced gastric cancer: a systematic review and meta-analysis based on aggregate data. J Clin Oncol. 2006;24(18): 2903-2909.

17. Vanhoefer U, Rougier $\mathrm{P}$, Wilke H, et al. Final results of a randomized phase III trial of sequential high-dose methotrexate, fluorouracil, and doxorubicin versus etoposide, leucovorin, and fluorouracil versus infusional fluorouracil and cisplatin in advanced gastric cancer: a trial of the European Organization for Research and Treatment of Cancer Gastrointestinal Tract Cancer Cooperative Group. J Clin Oncol. 2000;18(14):2648-2657.

18. Waters JS, Norman A, Cunningham D, et al. Long-term survival after epirubicin, cisplatin and fluorouracil for gastric cancer: results of a randomized trial. Br J Cancer. 1999;80(1-2):269-272.

19. Roth AD, Fazio N, Stupp R, et al. Docetaxel, cisplatin, and fluorouracil; docetaxel and cisplatin; and epirubicin, cisplatin, and fluorouracil as systemic treatment for advanced gastric carcinoma: a randomized phase II trial of the Swiss Group for Clinical Cancer Research. J Clin Oncol. 2007;25(22):3217-3223.

20. Van Cutsem E, Moiseyenko VM, Tjulandin S, et al. Phase III study of docetaxel and cisplatin plus fluorouracil compared with cisplatin and fluorouracil as first-line therapy for advanced gastric cancer: a report of the V325 Study Group. J Clin Oncol. 2006;24(31): 4991-4997.

21. Ajani JA, Moiseyenko VM, Tjulandin S, et al. Clinical benefit with docetaxel plus fluorouracil and cisplatin compared with cisplatin and fluorouracil in a phase III trial of advanced gastric or gastroesophageal cancer adenocarcinoma: the V-325 Study Group. J Clin Oncol. 2007;25(22):3205-3209.

22. Overman MJ, Kazmi SM, Jhamb J, et al. Weekly docetaxel, cisplatin, and 5-fluorouracil as initial therapy for patients with advanced gastric and esophageal cancer. Cancer. 2010;116(6):1446-1453.

23. Tebbutt NC, Cummins MM, Sourjina T, et al. Randomised, noncomparative phase II study of weekly docetaxel with cisplatin and 5-fluorouracil or with capecitabine in oesophagogastric cancer: the AGITG ATTAX trial. Br J Cancer. 2010;102(3):475-481.

24. Li CP, Chen JS, Chen LT, et al. A phase II study of weekly docetaxel and cisplatin plus oral tegafur/uracil and leucovorin as first-line chemotherapy in patients with locally advanced or metastatic gastric cancer. Br J Cancer. 2010;103(9):1343-1348.

25. De Gramont A, Figer A, Seymour M, et al. Leucovorin and fluorouracil with or without oxaliplatin as first-line treatment in advanced colorectal cancer. J Clin Oncol. 2000;18(16):2938-2947.

26. Guimbaud R BO, Rebischung C, et al. Planned interim analysis of the intergroup FFCD-GERCOR-FNCLCC-AERO phase III study comparing two sequences of chemotherapy in locally advanced or metastatic gastric cancers. J Clin Oncol. 2009;27(15S):4533.

27. Cunningham D, Starling N, Rao S, et al. Capecitabine and oxaliplatin for advanced esophagogastric cancer. $N$ Engl $\mathrm{J} \mathrm{Med}$. 2008;358(1):36-46.

28. Malik I, Bernal P, Byrd J. A phase I study of docetaxel, oxaliplatin, and capecitabine (DOC) as first-line therapy of patients with locally advanced or metastatic adenocarcinoma of stomach and GE junction. Cancer Invest. 2010;28(8):833-838
29. Andersen M, Schonnemann KR, Yilmaz M, Jensen HA, Vestermark LW, Pfeiffer P. Phase I study of docetaxel, oxaliplatin and capecitabine (TEX) as first line therapy to patients with advanced gastro-oesophageal cancer. Acta Oncol. 2010;49(8):1246-1252.

30. Dank M, Zaluski J, Barone C, et al. Randomized phase III study comparing irinotecan combined with 5-fluorouracil and folinic acid to cisplatin combined with 5-fluorouracil in chemotherapy naive patients with advanced adenocarcinoma of the stomach or esophagogastric junction. Ann Oncol. 2008;19(8):1450-1457.

31. Guimbaud R, Bouché O, Rebischung C, et al. Planned interim analysis of the intergroup FFCD-GERCOR-FNCLCC-AERO phase III study comparing two sequences of chemotherapy in locally advanced or metastatic gastric cancers. J Clin Oncol. 2009;27(15 Supp1): Abstract 4533 .

32. Boku N, Yamamoto S, Shirao K, et al. Randomized phase III study of 5-fluorouracil (5-FU) alone versus combination of irinotecan and cisplatin (CP) versus S-1 alone in advanced gastric cancer (JCOG9912). J Clin Oncol. 2007;25(18S):965.

33. Cassidy J, Saltz L, Twelves C, et al. Efficacy of capecitabine versus 5-fluorouracil in colorectal and gastric cancers: a meta-analysis of individual data from 6171 patients. Ann Oncol. 2011. [Epub ahead of print].

34. Kang YK, Kang WK, Shin DB, et al. Capecitabine/cisplatin versus 5-fluorouracil/cisplatin as first-line therapy in patients with advanced gastric cancer: a randomised phase III noninferiority trial. Ann Oncol. 2009;20(4):666-673.

35. Koizumi W, Narahara H, Hara T, et al. S-1 plus cisplatin versus S-1 alone for first-line treatment of advanced gastric cancer (SPIRITS trial): a phase III trial. Lancet Oncol. 2008;9(3):215-221.

36. Ajani JA, Rodriguez W, Bodoky G, et al. Multicenter phase III comparison of cisplatin/S-1 with cisplatin/infusional fluorouracil in advanced gastric or gastroesophageal adenocarcinoma study: the FLAGS trial. $J$ Clin Oncol. 2010;28(9):1547-1553.

37. Bang YJ, Van Cutsem E, Feyereislova A, et al. Trastuzumab in combination with chemotherapy versus chemotherapy alone for treatment of HER2-positive advanced gastric or gastro-oesophageal junction cancer (ToGA): a phase 3, open-label, randomised controlled trial. Lancet. 2010;376(9742):687-697.

38. Kang Y, Ohtsu A, Van Cutsem E, et al. AVAGAST: a randomized, double-blind, placebo-controlled, phase III study of first-line capecitabine and cisplatin plus bevacizumab or placebo in patients with advanced gastric cancer (AGC). J Clin Oncol. 2010;28(18S). Abstr 4007.

39. El-Rayes BF, Zalupski M, Bekai-Saab T, et al. A phase II study of bevacizumab, oxaliplatin, and docetaxel in locally advanced and metastatic gastric and gastroesophageal junction cancers. Ann Oncol. 2010;21(10):1999-2004.

40. Sun W, Powell M, O'Dwyer PJ, Catalano P, Ansari RH, Benson AB 3rd. Phase II study of sorafenib in combination with docetaxel and cisplatin in the treatment of metastatic or advanced gastric and gastroesophageal junction adenocarcinoma: ECOG 5203. J Clin Oncol. 2010;28(18):2947-2951.

41. Bramhall SR, Hallissey MT, Whiting J, et al. Marimastat as maintenance therapy for patients with advanced gastric cancer: a randomised trial. Br J Cancer. 2002;86(12):1864-1870.

42. Ueda S, Hironaka S, Boku N, Fukutomi A, Yoshino T, Onozawa Y. Combination chemotherapy with irinotecan and cisplatin in pretreated patients with unresectable or recurrent gastric cancer. Gastric Cancer. 2006;9(3):203-207.

43. Kunisaki C, Imada T, Yamada R, et al. Phase II study of docetaxel plus cisplatin as a second-line combined therapy in patients with advanced gastric carcinoma. Anticancer Res. 2005;25(4):2973-2977.

44. Giuliani F, Molica S, Maiello E, et al. Irinotecan (CPT-11) and mitomycin-C (MMC) as second-line therapy in advanced gastric cancer: a phase II study of the Gruppo Oncologico dell' Italia Meridionale (prot. 2106). Am J Clin Oncol. 2005;28(6):581-585. 
45. Kodera Y, Ito S, Mochizuki Y, et al. A phase II study of weekly paclitaxel as second-line chemotherapy for advanced gastric cancer (CCOG0302 study). Anticancer Res. 2007;27(4C): 2667-2671.

46. Park SH, Kim YS, Hong J, et al. Mitomycin C plus S-1 as second-line therapy in patients with advanced gastric cancer: a noncomparative phase II study. Anticancer Drugs. 2008;19(3):303-307.

47. Rosati G, Bilancia D, Germano D, et al. Reduced dose intensity of docetaxel plus capecitabine as second-line palliative chemotherapy in patients with metastatic gastric cancer: a phase II study. Ann Oncol. 2007;18(Suppl 6):vi128-vi132.

48. Schoennemann KR, Bjerregaard JK, Hansen TP, et al. Biweekly cetuximab and irinotecan as second-line therapy in patients with gastroesophageal cancer previously treated with platinum. Gastric Cancer. 2011. [Epub ahead of print].
49. Bang YJ, Kang YK, Kang WK, et al. Phase II study of sunitinib as second-line treatment for advanced gastric cancer. Invest New Drugs. 2010. [Epub ahead of print].

50. Ott K, Fink U, Becker K, et al. Prediction of response to preoperative chemotherapy in gastric carcinoma by metabolic imaging: results of a prospective trial. J Clin Oncol. 2003;21(24):4604-4610.

51. Shimada H, Okazumi S, Koyama M, Murakami K. Japanese Gastric Cancer Association Task Force for Research Promotion: clinical utility of (1) F-fluoro-2-deoxyglucose positron emission tomography in gastric cancer. A systematic review of the literature. Gastric Cancer. 2011;14(1):13-21.

\section{Publish your work in this journal}

Cancer Management and Research is an international, peer-reviewed open access journal focusing on cancer research and the optimal use of preventative and integrated treatment interventions to achieve improved outcomes, enhanced survival and quality of life for the cancer patient. The journal welcomes original research, clinical \& epidemiological studies, reviews \& evaluations, guidelines, expert opinion \& commentary, case reports \& extended reports. The manuscript management system is completely online and includes a very quick and fair peerreview system, which is all easy to use. Visit http://www.dovepress.com/ testimonials.php to read real quotes from published authors. 